\title{
Análisis del proceso de comunicación de la estrategia y su influencia en las políticas de calidad en la industria textil ecuatoriana: un estudio de caso
}

\section{Analysis of the communication process of the strategy and its influence on quality policies in the Ecuadorian textile industry: a case study}

Santiago Nájera, PhD.

Pontificia Universidad Católica del Ecuador, Ecuador

David Hernán Montenegro Moreno, MBA

Pontificia Universidad Católica del Ecuador, Ecuador

Fernando Gustavo Pérez Sisa

Universidad Central del Ecuador, Ecuador

María Auxiliadora Guerrero, MBA

Universidad Internacional del Ecuador, Ecuador

Autor para correspondencia: snnajera072@puce.edu.ec,dhmontenegro@yahoo.es, fjperez@uce.edu.ec, maguerrerobe@uide.edu.ec

Fecha de recepción: 04 de Agosto 2017 - Fecha de aceptación: 15 de Enero de 2018

\section{Resumen}

El proceso de comunicación de la estrategia en una empresa y su influencia en las políticas de calidad constituye un tema clave a ser estudiado. El método de estudio del caso es una alternativa válida para efectuar una investigación cualitativa que permita obtener una comprensión significativa del caso específico.

Palabras claves: comunicación; estrategia; método de estudio del caso

\begin{abstract}
The process of communication the business strategy and its influence on the quality policies is a key issue to be studied. The case study method is a valid alternative to carry out an exhaustive qualitative investigation that allows to obtain a significant understanding of the specific case.
\end{abstract}

Key Words: communication; strategy; case study method 


\section{Introducción}

Un método apropiada para el estudio concreto de un caso único es el método de estudio del caso(Yin, 2015). Método que se halla vinculado a la investigación netamente cualitativa y tiene un enfoque orientado al alcance de una comprensión significativa del caso, a través de la realización de un exhaustivo análisis. Método que vinculado en la presente casuística al proceso de comunicación de la estrategia de diferenciación y de su influencia en las políticas de calidad de la empresa objeto de estudio (Tsang, 2014).

La creación de la estrategia de una compañía constituye una representación de las respuestas de la administración a aspectos importantes tales como la concentración en un solo negocio o una diversificación (Thompson, Peteraf, Gamble, Strickland III, \& Jain, 2008). Sin embargo, es imprescindible que la estrategia creada sea comunicada de forma apropiada y efectiva dentro de la organización, ya que toda organización se encuentra en constante comunicación consigo misma y de manera directa o indirecta con su entorno, mostrando así una identidad y una cultura (Joan, 2001).

\section{Marco Teórico}

Existen varios conceptos, teorías, relacionadas con la comunicación, estrategia, la administración estratégica, la generación e implementación de la estrategia (Ghemawat, 2002). Todos estos aspectos se hallan relacionados entre sí en el sentido de que la generación e implementación de la estrategia constituyen el génesis de la estrategia de diferenciación para poder crear a corto y largo plazo una apropiada y sostenible ventaja competitiva. Situación que derivará en una mejora de la rentabilidad y el éxito empresarial, mediante el desarrollo de productos o servicios de calidad (Bonoma, 1984). Influyendo además de manera directa en las políticas de calidad, lo cual se plasma cuando se puede percibir la satisfacción del cliente y se evidencia un alto nivel de compromiso empresarial. Este compromiso debe estar enmarcado dentro de una cultura fuerte, es decir una cultura donde existe la presencia dominante de ciertos valores y métodos operativos profundamente arraigados que "regulan" el comportamiento de una compañía y el ambiente en el centro de trabajo (Thompson et al., 2008).

Para que los valores y principios empresariales relacionados con las políticas de calidad puedan ser correctamente difundidos dentro de la organización, es importante que la Alta Gerencia reitere constantemente los principios y valores a los miembros de la organización y les explique cómo se relacionan con su entorno de trabajo (Wheelen \& Hunger, 2012). Así mismo, es necesario que se recalque la importancia del rol del empleado dentro de su empresa, en el presente caso, recalcar el papel del operario y su repercusión en las políticas de calidad (Thompson et al., 2008).

En este sentido, la comunicación eficaz es una de las capacidades medulares de los líderes, pues forma parte del estilo de liderazgo (Bass, 1985; D’Alessio, 2010). Existen investigaciones empíricas que afirman que los buenos líderes son también comunicadores eficaces, por lo que se entiende que existiría una relación positiva entre la capacidad de comunicación y el desempeño del líder (Lussier \& Achua, 2015). 
En síntesis, el papel de la alta gerencia es preponderante en la comunicación y creación de la cultura organizacional(Radomska, 2014). Es así, que desde la cabeza parte la difusión correcta de los principios, valores y en el presente caso de las políticas de calidad, lo cual desemboca en la elaboración de productos de calidad sin fallas y que satisfagan los requisitos del cliente (Herrmann \& Nadkarni, 2014). A continuación, en la figura 1 se esquematiza dicho proceso:

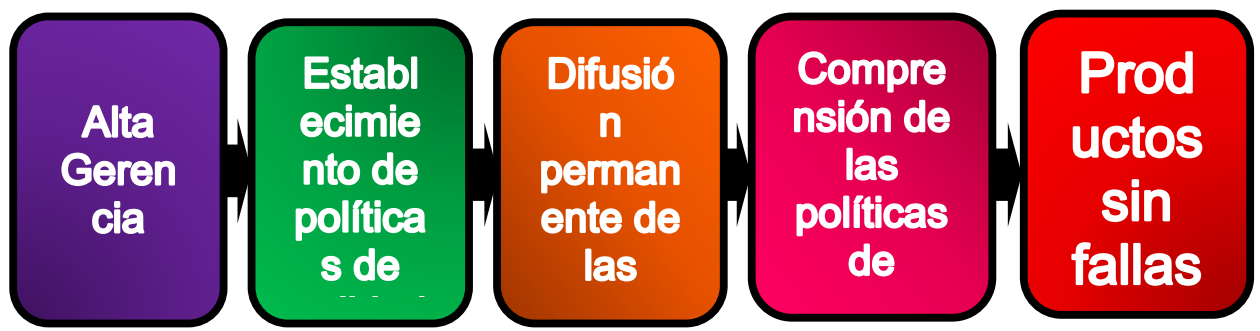

Figura 1. Esquema del proceso de comunicación

\section{Método de Investigación}

Cuando se utiliza el método de estudio del caso, una de las posibles fuentes de información a considerarse constituye la entrevista a profundidad semi-estructurada (Stake, 2010). En esta investigación corresponde la fuente primaria de información. Sin embargo, la información que se extrae de la misma corresponde a un proceso de múltiples recodificaciones de la información en búsqueda de obtener la esencia de la información obtenida, conforme se visualiza en la figura 2.
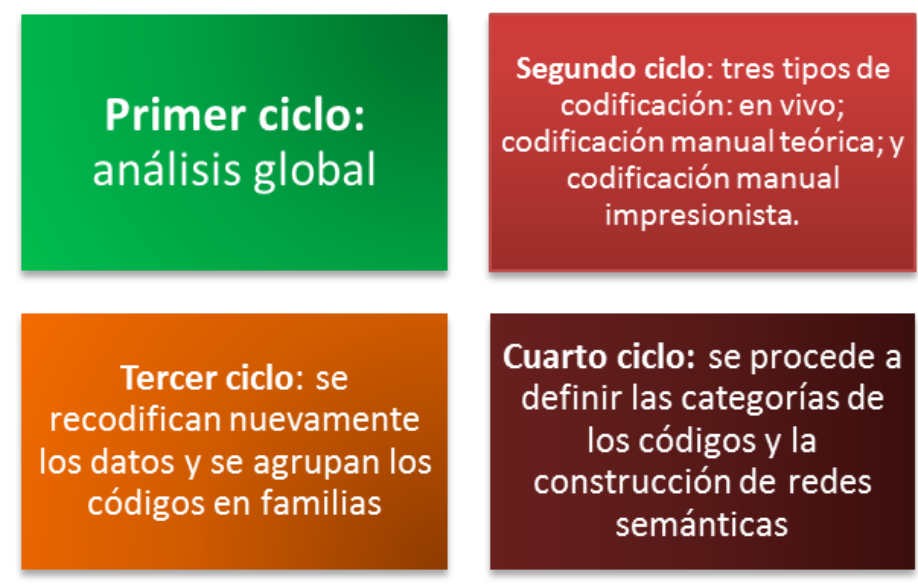

Quinto ciclo: con las redes

semánticas entorno a los

códigos y/o categorías, se

genera el modelo emergente

que busca describir la realidad

de los datos analizados.

Figura 2. Proceso de codificación y recodificación. 
Con la finalidad de efectuar una adecuada validación de los datos obtenidos es recomendable realizar la triangulación de datos (Tracy, 2012). Esta técnica permite verificar si el trabajo de investigación realizado, está dentro de los parámetros correctos (Gibbs \& Taylor, 2010). De esta forma, se asegura la validez de los resultados, contrastando con otro tipo de información.

\section{Resultados}

En el estudio efectuado en una empresa textil ecuatoriana, en donde se utilizó el método de estudio del caso, se evidenció que su estrategia es enfocada fundamentalmente en las políticas de calidad, es decir en otorgar productos de calidad que satisfagan enteramente las necesidades y requerimientos de los clientes.

Las estrategias se consolidan en el marco del consenso de un equipo multidisciplinario. Es decir, se evidenciaría que existe una adecuada comunicación entre las áreas, lo que fomenta el compromiso y la orientación hacia los objetivos. Esto se desprende de lo manifestado por los directivos entrevistados respecto de que las estrategias nacen de cada uno de los "cabezas de área", siempre orientadas a cumplir con los objetivos. La investigación revelo la existencia de una constante comunicación entre las áreas y hacia el resto de personal para generar la estrategia, privilegiando la experiencia y el conocimiento de cada integrante del equipo multidisciplinario.

Es factible acotar, que a partir de la Alta Gerencia se establece la estrategia, influyendo ésta en las políticas de calidad. Sin embargo, para asegurar la obtención de un producto final sin fallas, resulta necesario efectuar una permanente difusión de las políticas de calidad entre los empleados a fin de comprometerlos con la calidad: es decir, una comunicación continua.

\section{Conclusiones}

Si bien, los resultados que se obtienen de la investigación, al tratarse de un caso único, no son generalizables, sí es factible obtener una comprensión significativa del caso. El estudio revela que la comunicación continua al interior de la empresa y la retroalimentación entre mandos medios y la alta gerencia permiten el financiamiento de las políticas de calidad. Políticas que responden a la estrategia propia de la empresa, y que se convierten en su factor diferenciador.

\section{Bibliografía}

Bass, B. M. (1985). Leadership and performance beyond expectations: Free Press; Collier Macmillan.

Bonoma, T. V. (1984). Making your marketing strategy work. Harvard Business Review, 62(2), 69-76.

D’Alessio, F. A. (2010). Liderazgo y atributos gerenciales: una visión global y estratégica: PEARSON. 
Ghemawat, P. (2002). Competition and business strategy in historical perspective. Business history review, 76(01), 37-74.

Gibbs, G. R., \& Taylor, C. (2010). How and what to code. Online QDA.

Herrmann, P., \& Nadkarni, S. (2014). Managing strategic change: The duality of CEO personality. Strategic Management Journal, 35(9), 1318-1342.

Joan, C. (2001). Imagen Corporativa en el siglo XXI. Ediciones la Crujia, Buenos Aires-Argentina.

Lussier, R. N., \& Achua, C. F. (2015). Leadership: Theory, application, \& skill development: Nelson Education.

Radomska, J. (2014). Linking the Main Obstacles to the Strategy Implementation with the Company's Performance. Procedia - Social and Behavioral Sciences, 150(0), 263-270. doi:http://dx.doi.org/10.1016/j.sbspro.2014.09.054

Stake, R. (2010). Qualitative Research: Studying How Things Work: Guilford Publications.

Thompson, A., Peteraf, M., Gamble, J., Strickland III, A. J., \& Jain, A. K. (2008). Crafting \& Executing Strategy 19/e: The Quest for Competitive Advantage: Concepts and Cases: McGraw-Hill Education.

Tracy, S. J. (2012). Qualitative Research Methods: Collecting Evidence, Crafting Analysis, Communicating Impact: Wiley.

Tsang, E. W. K. (2014). Case studies and generalization in information systems research: A critical realist perspective. The Journal of Strategic Information Systems, 23(2), 174-186. doi:10.1016/j.jsis.2013.09.002

Wheelen, T. L., \& Hunger, J. D. (2012). Strategic Management and Business Policy: Toward Global Sustainability: Pearson Prentice Hall.

Yin, R. K. (2015). Qualitative Research from Start to Finish: Guilford Publications. 